\title{
Mathematical Indispensability and Arguments from Design
}

\author{
Silvia Jonas ${ }^{1}$ \\ Received: 5 November 2020 / Revised: 16 February 2021 / Accepted: 26 February 2021 / \\ Published online: 16 April 2021 \\ (C) The Author(s) 2021
}

\begin{abstract}
The recognition of striking regularities in the physical world plays a major role in the justification of hypotheses and the development of new theories both in the natural sciences and in philosophy. However, while scientists consider only strictly natural hypotheses as explanations for such regularities, philosophers also explore meta-natural hypotheses. One example is mathematical realism, which proposes the existence of abstract mathematical entities as an explanation for the applicability of mathematics in the sciences. Another example is theism, which offers the existence of a supernatural being as an explanation for the design-like appearance of the physical cosmos. Although all meta-natural hypotheses defy empirical testing, there is a strong intuition that some of them are more warranted than others. The goal of this paper is to sharpen this intuition into a clear criterion for the (in)admissibility of meta-natural explanations for empirical facts. Drawing on recent debates about the indispensability of mathematics and teleological arguments for the existence of God, I argue that a meta-natural explanation is admissible just in case the explanation refers to an entity that, though not itself causally efficacious, guarantees the instantiation of a causally efficacious entity that is an actual cause of the regularity.
\end{abstract}

Keywords Cosmic fine-tuning - Arguments from design · Mathematical realism · Indispensability argument $\cdot$ Mathematical explanation

\section{The Legitimacy Of Meta-Natural Hypotheses}

The recognition of striking order, patterns, and correlations lies at the heart of the natural sciences and is a core element of scientific progress. It often receives its initial impetus through reasoning by analogy, an indispensable heuristic tool in the development of new theories that enables the extraction of general categories and

Silvia Jonas

silvia.jonas@1mu.de

1 Munich Center for Mathematical Philosophy (MCMP), Munich University, Munich, Germany 
rules from complex patterns, lends justification to new hypotheses by relating our past experience to new problems in order to demonstrate parallels with already accepted hypotheses, and provides a basis for the unification of theories about distinct phenomena. ${ }^{1}$

When scientists observe unexplained, striking regularities, they begin to investigate possible explanations. However, not all logically possible explanations count as legitimate objects of scientific study. For example, in order to explain the spiralshaped arrangement of seeds in sunflower heads, a biologist would not consider the hypothesis that the seeds were arranged by beauty-loving angels. Rather, natural scientists investigate only strictly natural hypotheses. ${ }^{2}$

This is different with philosophers. The investigation of striking order, patterns, and correlations also plays a major role in philosophy, but philosophers don't restrict their attention to empirical hypotheses. Rather, they also develop 'meta-natural' explanations, i.e. explanations that transcend the realm of scientific investigation. For example, moral realists posit the existence of abstract, non-observable moral entities (such as reasons or values), which some even describe as having quasi-causal powers. Typically, meta-natural hypotheses come into play when empirical methods offer no suitable way of investigating a striking order, pattern, or correlation (below I introduce four classical cases). Such meta-natural hypotheses are then judged according to theoretical criteria such as explanatory power, internal consistency, coherence with accepted philosophical views, etc.

However, no matter how well they meet such theoretical criteria, not all metanatural hypotheses are considered prima facie equally legitimate. Some appear to enjoy more initial credibility than others. For example, no matter how much explanatory power the hypothesis of theism has, many philosophers do not consider the existence of God a legitimate explanation for striking empirical regularities. By contrast, many philosophers and mathematicians are perfectly happy to subscribe to some form of mathematical realism, i.e. the view that mathematical statements are about mind-independent mathematical truths and have objective, determinate truth-values, even if this evidently implies some kind of abstract, 'non-natural' mathematical ontology. This seems like a double-standard. After all, given that both the existence of God and the existence of numbers defy empirical confirmation, both hypotheses ought to be considered prima facie equally legitimate.

\footnotetext{
${ }^{1}$ See Bartha (2010) for a thorough investigation of the formal characteristics of analogical reasoning. For an excellent discussion of the role of analogical reasoning in major breakthroughs in $18^{\text {th }}$ and $19^{\text {th }}$ century physics, see Steiner $(1989,1998)$.

${ }^{2}$ What counts as natural is not always clear and may change over time. For example, when Newton formulated his theory of gravity, which explains both terrestrial and celestial motions in terms of an unobservable force acting at a distance across empty space, Leibniz condemned this as positing an 'occult quality,' an illegitimate meta-natural explanation. From today's point of view, calling gravity a meta-natural posit seems absurd. However, now there are a number of new scientific hypotheses - say, the multiverse—whose status as natural or meta-natural hypothesis is unclear. Thus, the boundaries between the natural and the meta-natural may be fluid, yet as will become clear below, this paper is concerned with clear-cut cases of meta-natural posits only.
} 
In fact, however, there is a strong intuition that some meta-natural explanations are prima facie more warranted than others. The way this intuition is usually substantiated is by reference to the particular features of the entities in question and the problems these features raise in a particular context. For example, most metaphysicians reject Lewisian modal realism, according to which there exist infinitely many concrete yet causally disconnected possible worlds. The reason they reject this view is of course not that the view failed empirical testing. Rather, besides its counterintuitiveness and its vastly inflated ontology, modal realism raises a number of issues that are problematic within the greater context of the philosophy of modality, for example Kaplan's paradox ${ }^{3}$ or the problem of island universes. ${ }^{4}$

The goal of this paper is to sharpen the intuition that some meta-natural hypotheses are more warranted than others into a clear criterion for the (in)admissibility of meta-natural explanations of empirical facts. I adopt the criterion that a meta-natural explanation is admissible just in case the explanation refers to an entity that, though not itself causally efficacious, guarantees the instantiation of a causally efficacious entity that is an actual cause of the regularity. I show that only the theism hypothesis, but not the multiverse and the chance hypotheses meets the basic criteria for a program explanation.

\section{Meta-Natural Explanations of Empirical Regularities}

Here are four examples of meta-natural hypotheses that have been offered in a variety of philosophical contexts in order to explain particular kinds of order, patterns, or correlations observable in the physical world. I have sorted the examples in descending order of intuitive 'acceptability:'

Mathematical entities: Ancient Greek philosophers like Pythagoras, Plato, and Euclid transformed mathematics - hitherto nothing but a tool for the solution of practical problems - into an abstract science whose clarity, precision, and rigour soon became a standard for all other sciences. However, this abstract conception of mathematics, according to which mathematical properties supervene on mathematical, rather than physical entities, also raises profound philosophical questions. For example, how can a purely abstract system be applicable to the empirical world (and be incredibly successful at at that)? As Wigner puts it: 'the enormous usefulness of mathematics in the natural sciences is something bordering on the mysterious' (Wigner 1960, p. 2). One way to explain this correlation between our

\footnotetext{
${ }^{3}$ Assume that propositions are sets of possible worlds and that there are k possible worlds. Each subset of the set of all possible worlds constitutes a proposition. A set of cardinality $\mathrm{k}$ has $2^{k}$ subsets. $2^{k}$ is strictly greater than $\mathrm{k}$. For every proposition, there is a possible world in which it constitutes the sole content of a subject's thought. Hence, there are at least $2^{k}$ possible worlds. Contradiction. Cf. Kaplan (1995).

${ }^{4}$ Lewis introduces possible worlds in order to provide a semantics for modal statements. However, in order to characterise the totality of possible worlds as a collection of distinct worlds rather than parts of a single disjoint world, one needs to presuppose modal notions, such as the notion of a 'worldmate.' This, then, seems viciously circular. Cf. Bigelow and Pargetter (1987).
} 
mathematical and empirical facts is to endorse 'mathematical Platonism,' a philosophical view at the core of which stands the meta-natural hypothesis that there exist irreducibly abstract, mind-independent mathematical entities. Plato (1997) argues that the correlation between abstract entities ('Forms' or 'Ideas') and their physical counterparts is due to 'participation:' all particulars falling under a certain predicate 'participate' in the Form denoted by that predicate. More recently, the view that Platonism best explains the applicability of mathematics to science has been discussed in the context of 'enhanced indispensability arguments' (EDAs), whose central claims are (a) that we should be ontologically committed to all parts of a scientific theory that make a genuine explanatory contribution to the explanation of a physical phenomenon, and (b) that there are cases where mathematical entities play precisely such a role..$^{5}$ As Baker 2011, p. 266 writes,

The challenge to explain the explanatory effectiveness of mathematics in science is one that can only be adequately met by a realist view of mathematics such as platonism. This is especially true if we combine the indispensability issue with the explanation issue and ask why mathematics plays an indispensable explanatory role in science. Compare this with a corresponding question for theoretical concrete entities. Why are electrons explanatorily indispensable? The natural response here is: because electrons exist! To try [n.b.: as nominalists do] to explain a phenomenon by reference to acknowledged fictions leads to a situation of rational instability. Thus considering the question of why mathematical entities play an explanatory role in science may provide the best route to a compelling defence of mathematical platonism.

Moral entities and properties: Also metaethicists have offered meta-natural hypotheses to explain empirical regularities. For instance, robust moral realism, i.e. the view according to which there exist objective, mind-independent moral entities (e.g. reasons and values) and properties (e.g. rightness or wrongness), has been suggested as an explanation for the empirical fact that people tend to take moral matters seriously, or more specifically, that in the face of moral disagreement, they tend to reason and act in ways analogous to the ones we use to resolve disagreements about strictly empirical facts. ${ }^{6}$

Possible worlds and propositions: As mentioned above, meta-natural explanations of empirical regularities also feature in metaphysics. The most notorious example are possible worlds (and propositions, possible worlds' sidekickspropositions are frequently defined as sets of possible worlds, and possible worlds are even more frequently defined in terms of sets of propositions). In order to

\footnotetext{
${ }^{5}$ Cases of allegedly indispensable mathematical explanations in science include the Magicicada, which will be discussed below; the falling pattern of sticks thrown into the air (Lipton 2004); the crossing of bridges at Königsberg (Pincock 2007); the geometrical properties of Minkowski space-time as exemplified in the bending of light near massive bodies, and in the Lorentz-FitzGerald contraction of moving bodies in special relativity (Colyvan 2001; 2002); the location of the Kirkwood gaps (Colyvan 2010); the hexagonal shape of honeycomb cells (Lyon and Colyvan 2008; Lyon 2012); the spiral arrangement of sunflower seeds (Lyon 2012); and Plateau's laws for soap films (Lyon 2012; Pincock 2015).

${ }^{6}$ See, for example, Enoch (2011), p. 23f.
} 
explain the fact that our reasoning about what is and isn't possible is so extraordinarily useful across a wide range of different contexts, modal realists have proposed the hypothesis that the modal statements employed in such reasoning are true of concrete physical worlds that are spatiotemporally and causally disconnected from our actual world, but in all other respects just like our actual world. $^{7}$

God: The oldest (and least accepted by Western philosophers) meta-natural hypothesis is theism, which posits the existence of a cosmic creator, conventionally referred to as 'God.' Different versions of this hypothesis have been offered for thousands of years as an explanation for various kinds of regularities in the empirical world. Such 'arguments from design,' or 'teleological arguments,' begin with a premise that points out some type of order observable in the physical cosmos, argue that this type of order would not exist had it not been intentionally created, and end with a conclusion that proclaims the (likely) existence of an intelligent being or 'designer' who created the physical cosmos. ${ }^{8}$ Despite the fact that the kinds of order or regularity that are relevant for arguments from design have been a topic of philosophical discussion for over two thousand years, ${ }^{9}$ and despite the fact that many of the phenomena once thought to cry out for metanatural explanation can now be explained in purely scientific terms, ${ }^{10}$ and despite the fact that no formulation of the design argument has yet been found that is accepted by all participants in the debate, ${ }^{11}$ design arguments continue to persist, and even philosophers who would reject theistic conclusions of any sort admit that the intricate functional organisation of our cosmos is striking.

Generally speaking, though, (Western) philosophers are much more comfortable positing the existence of mathematical entities or truths than they are with positing the existence of God. Is there any domain-independent fact, i.e. a fact independent of the particular objections occurring in each of the individual debates, that can account for our different intuitions regarding meta-natural hypotheses?

In order to answer this question, I now turn to the current debate about mathematical realism and arguments from explanatory indispensability cashed out in terms of the concept of 'program explanation.' The concept of program explanation illuminates how mathematics can play a genuinely explanatory role in our best scientific theories. Drawing an analogy with the case of theism, I argue that positing the existence of God as a meta-natural explanation for cosmic fine-tuning is a prima facie admissible hypothesis. However, I also argue that much more work needs to be done

\footnotetext{
${ }^{7}$ The classic account of modal realism is of course (Lewis 1986).

${ }^{8}$ Comprehensive introductions to Design Arguments are Sedley (2007) (focusing on discussions from Antiquity), Jantzen (2014) (covering arguments from Antiquity to Modernity), and Sober (2018).

${ }^{9}$ The versions that are currently considered to have most (intuitive) force are fine-tuning arguments. These arguments start with a premise according to which the universe is evidently fine-tuned for the existence of life, and that the probability of the laws of physics corresponding exactly to the conditions necessary to enable life is incredibly small; cf. Friederich (2018).

${ }^{10}$ Think about meteorological phenomena like thunderstorms or rainbows, astronomical phenomena like comets or solar eclipses, geological phenomena like earthquakes, etc.

${ }^{11}$ Design Arguments are standardly classified as analogical arguments, though there have been efforts to give them a deductive or abductive (IBE) structure. See, for example, Ratzsch and Koperski (2019).
} 
in order to show that the God-hypothesis can play an indispensable programming role analogous to the mathematical case, and even if this work could be done, only a very thin/ deflated notion of God could be shown to be warranted (perhaps even only a designer, who might as well be a demon). But this is an important result nevertheless, given that it shows us what fine-tuning arguments can potentially achieve (i.e. establish existence of designer), and what they can never achieve (establish existence of God with classical divine attributes).

\section{Mathematical Explanations of Empirical Facts}

For some time now, debates about mathematical realism have focused on issues concerning how best to understand mathematical explanation. At the centre of these discussions is the question whether, at least in some specific cases, mathematics plays an explanatorily indispensable role in the scientific explanation of particular empirical phenomena. Mathematical realists argue that the scientific explanations of those phenomena contain mathematical elements whose role in the scientific explanation cannot be reduced to a mere representation of empirical regularities. Here are three examples:

Honeycombs: Bees build their honeycombs out of hexagonal cells. This striking fact calls for explanation. Darwin argued that minimising the amount of energy and wax used for the construction of honeycombs generates an evolutionary advantage, such that the bees who are most efficient in the use of energy and wax will be selected (Darwin 1859). In 1999, Thomas Hales proved that 'a hexagonal grid is the most efficient way to divide a Euclidean plane into regions of equal area with least total perimeter' (Hales 2001, p. 4).

Cicadas: Certain types of North American cicada, Magicicadas, have primenumbered life cycles. They emerge from the ground every 13 and 17 years. This striking fact calls for explanation. Due to a number of ecological constraints, for example the need to minimise intersection with periodic predators with different cycle periods, having a prime-numbered life-cycle is advantageous for the Magicicada (Baker 2005, 2009; Goles et al. 2001, p.33). The reason for this is the number-theoretic fact that prime numbers maximise their lowest common multiple relative to all lower numbers. ${ }^{12}$

Sunflower seeds: Sunflowers arrange their seeds in a striking spiral pattern. The explanation for this is again a combination of evolutionary with mathematical facts. Fitting as many seeds as possible into the circular flowerhead constitutes an evolutionary advantage. Sunflowers grow their seeds at the centre of the flowerhead, with new seeds pushing older ones outwards. Whenever a new seed develops, it does so at some angle of rotation from the older one. Given this way of growing seeds, the optimal rotation angle, i.e. the rotation angle at which the highest amount of seeds can be fitted into the flowerhead, can be made mathematically precise: it is an irrational fraction of 360 degrees, roughly 137.5 degrees,

\footnotetext{
${ }^{12}$ For proofs of the relevant lemmas, see (Landau 1958).
} 
which is equivalent to the complement of $360 \varphi \bmod 360$ (where $\varphi$ is the Golden Ratio; Lyon 2012, p. 4).

What distinguishes those examples from other scientific theories featuring mathematics is that the mathematical part of the explanation plays an explanatory role of its own, i.e. it constitutes part of the explanation of the empirical phenomenon. Moreover, it plays this role indispensably, i.e. there is no way of explaining the empirical phenomenon without reference to the respective mathematical facts.

The fact that mathematics is explanatorily indispensable to some of our best scientific theories can then be argued to support mathematical realism: if we believe that we should be ontologically committed to all and only those parts of a scientific theory that are explanatorily indispensable, i.e. that contribute to the explanation of the physical phenomenon the scientific theory aims to explain, then the fact that mathematical entities play this role in some-perhaps many-scientific theories commits us to the belief in the existence of mathematical entities, or at least, renders the belief in the existence of those entities pro tanto admissible. ${ }^{13}$

The question now is: Could it be possible for the meta-natural posit conventionally referred to as 'God' to play an analogously indispensable role in the explanation of empirical regularities, such that, once we recognise this role, we are committed to believe in the existence of this entity? In order to answer this question, we need to develop a more precise idea of what exactly makes the explanations in the above example mathematical, i.e. how exactly the mathematics featuring in the above examples explains the empirical facts in question.

\section{Explanations Featuring Mathematics}

The natural sciences are full of mathematics: it is an indispensable tool for the formulation of theories about the physical world. In many of those theories, the mathematics involved plays a merely representational role, for example by representing quantities. However, the mathematical elements involved in the examples above are themselves an integral part of the explanation: Hales' theorem explains why bees build their honeycombs out of hexagonal cells; the number-theoretic properties of prime numbers explain the length of the life-cycles of Magicicadas; and the irrational number equivalent to the Golden Ratio explains the elegant arrangement of sunflower seeds. How exactly is it possible for a purely mathematical, i.e. causally inert fact to explain a strictly empirical regularity? To answer this question, let's look at two kinds of explanation of empirical regularities that involve mathematics without it playing an explanatory role.

\footnotetext{
${ }^{13}$ The original indispensability argument, according to which all parts of a scientific theory get confirmed when the theory as a whole is confirmed, is of course due to Quine (1981) and Putnam (1979). However, because of the much criticised 'confirmational holism' implicit in their version of the argument, the new, 'enhanced' form of the indispensability argument (Baker 2005; 2009; Colyvan 2001) now requires that an entity play an explanatorily indispensable role in a scientific theory.
} 


\subsection{Empirical Instantiations of Mathematical Truths}

It is a theorem of mathematics that the sum of the internal angles of any triangle in a Euclidean space is 180 degrees. This mathematical truth is instantiated in every physically existing triangle in a Euclidean space (setting aside unavoidable discrepancies between mathematical ideals and corresponding physical approximations). However, it does not explain anything about why some particular physical triangle, for example a set square, exists; such an explanation would involve some story about how a chain of physical events led to the creation of a physical triangle.

\subsection{Mathematical Representations of Empirical Facts}

Einstein's mass-energy equivalence $E=m c^{2}$ states that the energy $E$ of any entity with a mass is equivalent to its mass $m$ multiplied by the speed of light $c$ squared. Clearly, this equation is formulated with the help of mathematical language ('=', ' 2 '). However, the variables and constants featuring in it represent physical quantities: an object's mass (m) and energy (E) as well as the speed with which light travels (c); the equation employs mathematical language for the sole purpose of representing physical relations in a convenient and economical way. Hence, even though it clearly features mathematics, the mathematical elements do not contribute to the explanation of the relations holding between the physical properties and objects.

\subsection{Mathematical Explanations of Empirical Facts}

So how exactly is it possible for a purely mathematical fact to explain an empirical regularity? What distinguishes the honeycomb, the Magicicada, and the sunflower examples from mere instantiations of mathematical truths in the physical world on the one hand, and from explanations using mathematical language for purely representational purposes on the other?

A theory introduced by Jackson and Pettit (1990) in the context of causal explanations, and later applied to mathematical explanations by Lyon (2012) provides a plausible answer: Mathematical properties featuring in mathematical explanations of empirical phenomena, though not causally efficacious, are causally relevant to the empirical phenomena in question by programming for the instantiation of the phenomenon. This needs some unpacking.

Let's begin with Jackson and Pettit. The central idea behind their account of 'program explanations' is that causal explanations of physical phenomena need not involve reference to causally efficacious properties.

A causally efficacious property with regard to an effect [i.e. a physical phenomenon] is a property in virtue of whose instantiation, at least in part, the effect occurs; the instance of the property helps to produce the effect and does so because it is an instance of that property... A property $\mathrm{F}$ is not causally efficacious in the production of an effect $e$ if these three conditions are fulfilled together. 
(i) there is a distinct property $\mathrm{G}$ such that $\mathrm{F}$ is efficacious in the production of $e$ only if $\mathrm{G}$ is efficacious in its production [co-instantiated];

(ii) the F-instance does not help to produce the G-instance in the sense in which the $\mathrm{G}$-instance, if $\mathrm{G}$ is efficacious, helps to produce $e$; they are not sequential causal factors [non-sequential];

(iii) the F-instance does not combine with the G-instance, directly or via further effects, to help in the same sense to produce $e$ (nor of course, vice versa): they are not coordinate causal factors [non-coordinate]. (Jackson and Pettit 1990, p. 108)

Causally efficacious properties thus contribute directly to the production of the physical phenomenon in question. For example, since an object of constant mass accelerates in proportion to the force applied to it, a causal explanation of the speed of a tennis ball at a given time $t$ will refer to the force $\mathrm{F}$ acting on the ball when it was hit. The ball's mass and the force acting on it are causally efficacious properties because they contribute directly to the production of the physical phenomenon in question, i.e. the speed of a tennis ball at a given time $t$.

Now, it is plausible to assume that causal explanations of physical phenomena must only invoke causally relevant (as opposed to causally irrelevant) properties. However, as Jackson and Pettit show, it is implausible to assume that causally relevant properties are necessarily causally efficacious properties. The structure of their argument is as follows:

1. Assume that all causal explanations invoke only causally relevant properties.

2. Assume that all causally relevant properties are causally efficacious.

3. Causal explanations thus invoke only causally efficacious properties.

4. Causally efficacious properties contribute directly to the production of X; they do not fulfil conditions (i), (ii), and (iii).

5. The properties invoked in explanations of physical phenomena in basic science, i.e. physics (e.g. 'having mass $X$ ' or 'having positive charge') do not fulfil conditions (i), (ii), and (iii); hence, they are causally efficacious.

6. Explanations of physical phenomena in the special sciences, e.g. sociology, psychology or biology, invoke properties like 'the property of a group that it cohesive; of a mental state that it is the belief that p; of a biological trait that it maximizes inclusive fitness' (Jackson and Pettit 1990, p. 112).

7. The properties invoked in explanations of physical phenomena in the special sciences are not causally efficacious because they fulfil conditions (i), (ii), and (iii).

8. Conclusion: Explanations of physical phenomena in the special sciences are not causal explanations.

The conclusion that causal explanations are only to be found in basic science but not in the special sciences is, of course, absurd. The most plausible and straightforward way to avoid this conclusion is to resist premise (2), i.e. the assumption that causally relevant properties must be causally efficacious. In order to resist that 
assumption, what needs to be shown is that it is possible for a causally inert property to be causally relevant to the production of a physical phenomenon. Jackson and Pettit use the following example to demonstrate this possibility.

Question: What explains that a piece of uranium emits radiation over a certain period? Answer: The property of the uranium that some of its atoms were decaying. Note that this answer involves existential quantification over some of the uraniums atoms, though not over such and such particular atoms. The property invoked in the explanation of the radioactivity of a piece of uranium is thus an abstract, higherorder property. However, higher-order properties fulfil conditions (i) - (iii): they are co-instantiated with other, causally efficacious properties, yet they relate to those in a non-sequential and non-coordinate manner. Higher-order properties are thus not causally efficacious. If the property of the uranium that that some of its atoms were decaying was efficacious, then only because the lower-order property that such and such particular atoms were decaying was efficacious. However, the higher-order property of the uranium that some of its atoms were decaying is the one that features in the causal explanation of the radioactivity of uranium. Thus, the higher-order property, though causally inert, is clearly causally relevant. How is this possible? Here is Jackson's and Pettit's answer:

\begin{abstract}
Although not efficacious itself, the [higher-order] property was such that its realization ensured that there was an efficacious property in the offing: the property, we may presume, involving such and such particular atoms. The realization of the higher-order property did not produce the radiation in the manner of the lower-order. But it meant that there would be a suitably efficacious property available, perhaps that involving such and such particular atoms, perhaps one involving others. And so the property was causally relevant to the radiation, under a perfectly ordinary sense of relevance, though it was not efficacious. It did not do any work in producing the radiation - it was perfectly inert - but it had the relevance of ensuring that there would be some property there to exercise the efficacy required. (Jackson and Pettit 1990, p. 114)
\end{abstract}

The higher-order property thus ensures, without being itself part of the productive process leading to the empirical phenomenon, that the crucial, physically productive property is realised and the empirical phenomenon occurs. As a description of the relationship between such a property and an effect Jackson and Pettit choose the metaphorical term 'programming', which evokes the analogy with a computer program ensuring that certain events will occur, even though all of the actual physical work of producing them goes on at a lower, mechanical level. Their theory thus carves out an important distinction between two ways in which properties can play an explanatory role in empirical theories:

It appears then that there are at least two distinct ways in which a property can be causally relevant: through being efficacious in the production of whatever is in question, or through programming for the presence of an efficacious property.' (Jackson and Pettit 1990, p. 115) 
This distinction enables us to explain what makes explanations in the special sciences causally relevant, even though they are not causally efficacious: higherorder properties, for example 'maximizing inclusive fitness,' program for particular lower-order properties, for example 'having strong teeth', which in turn contribute to the survival of individual species. In fact, it is plausible to assume that, perhaps with the exception of physics, most of the explanations scientists offer for empirical phenomena are 'program' rather than 'process' explanations.

As Lyon (2012) observes, the distinction between two kinds of causally relevant properties applies not only to physical, but also to mathematical explanations of empirical phenomena. Consider Putnam's classic peg-hole example (Putnam 1975, pp. 295ff). We imagine a wooden board with two holes, one circular with a diameter of one inch, the other square with a side-length of one inch. What explains the fact that a cubical peg with a side-length of 15/16ths of an inch on each side will fit through the square hole but not the round hole? The answer to this question will most certainly invoke mathematical properties. For example, we might say that any peg with a side-length of 15/16ths of an inch is too large for any hole with a one-inch diameter.

Strictly speaking, the properties that are efficacious in causing the peg to bump into the board rather than pass through the hole are the peg's micro-physical properties, such as its spatiotemporal coordinates, the forces acting on the bodies, their molecular structure, fundamental components, etc. However, there is a strong sense in which the peg's micro-physical properties provide only part of the explanation of the peg's failure to pass through the hole. A full explanation would also mention the peg's and the board's geometrical properties respectively. Using Jackson's and Pettit's terminology, the micro-physical properties of peg and board are causally relevant to the peg's failure to pass the board by being efficacious in the production of the 'bump;' the geometrical properties of peg and board are causally relevant because they program for the presence of the relevant micro-physical properties.

Also the honeycombs, the Magicadas, and the sunflower seeds can be analysed in this way: the explanation of the respective empirical regularities (hexagonal cell shapes, prime-numbered life-cycles, Golden Ratio rotation angles) involve a purely mathematical element as well as a causal element that works on the micro-physical level.

What distinguishes empirical instantiations of mathematical truths as well as mathematical representations of empirical facts from mathematical explanations is thus the way in which the mathematical element of an hypothesis contributes to the explanation of the empirical phenomenon in question, i.e. by playing a programming role. Through this role, it is possible for a purely mathematical fact to be causally relevant in the explanation of an empirical regularity. And if, at least sometimes, mathematics plays the programming role indispensably, it is admissible-perhaps even necessary-to draw ontological conclusions from this fact, i.e. to posit the existence of mathematical entities (Baker 2005; 2009; Colyvan 2001).

In the final part of the paper, we will now turn to the case of theism and see how the programming account fares there. 


\section{Theistic Explanations of Empirical Facts}

The question we raised earlier was: Could it be possible for the meta-natural posit conventionally referred to as 'God' to play an analogously indispensable programming role in the explanation of empirical regularities, such that, once we recognise this role, it is prima facie legitimate for us to believe in the existence of such an entity?

Theistic explanations of empirical regularities as they feature in arguments from design can be plausibly understood as attempts to establish precisely that. One of the earliest arguments from design can be found in Cicero's The Nature of the Gods:

If the first sight of the universe happened to throw [philosophers] into confusion, once they observed its measured, steady movements, and noted that all its parts were governed by established order and unchangeable regularity, they ought to have realised that in this divine dwelling in the heavens was one who was not merely a resident but also a ruler, controller, and so to say the architect of this great structural project. (Cicero 1998, II.90, p. 79; see also Jantzen 2014, p. 37)

Since Cicero's times, countless variations of the argument from design have been suggested, but their main structure is always roughly like this:

1. We perceive regularities (of some striking kind) in the physical world.

2. There is no plausible way of explaining these independent of deliberate intent.

3. Deliberate intent implies a designer.

4. The designer is God.

Arguments with this structure can be attacked in different ways, depending on which premise is considered implausible. However, premise 2 is the one that has come under attack most frequently, viz. whenever scientists developed purely natural explanations for phenomena that, at one point, seemed to call for supernatural explanations. And this makes sense, of course: The more empirically tractable allegedly design-like properties are, the less we accept them as actual marks of design and purpose. Despite the many successes of empirical science, however, attempts to account for striking regularities in theistic terms never completely vanished from the philosophical landscape. In fact, some versions of the design argument, most notably the argument from cosmic fine-tuning, have drawn a lot of attention lately and have developed the argument in great detail. ${ }^{14}$

I will now briefly outline the main structure of fine-tuning arguments. I will then apply the concept of 'program' explanation in order to investigate whether the designer-hypothesis plays an indispensable programming-role in those arguments, such that recognition of this role makes it prima facie legitimate to posit the existence of God.

Fine-tuning arguments cash out premise 1, i.e. the observation that there are striking regularities in the empirical world, in terms of (a) the fine-tuning of the physical cosmos, more precisely, the fact that the universe appears to be functionally organised

\footnotetext{
${ }^{14}$ See, for example, Holder (2002).
} 
in a way that makes life possible, and (b) the improbability of the physical constants falling exactly into the range required for the development of life.

For example, if the cosmological constant $\Lambda$, the parameter representing the expansion rate of the universe, were only slightly smaller than it is, then the universe would have collapsed back onto itself shortly after the Big Bang. If $\Lambda$ were only slightly greater than it is, stars could not have developed. And since stars are the only known sources in the universe capable of producing large quantities of the elements on which all living organisms crucially depend-oxygen, carbon, hydrogen, etc.life without stars would arguably not be possible. Taking into consideration all of the fine-tuning examples relevant to the formation of stars, the chance of stars existing in the universe has been estimated by theoretical physicists to be 1 in $10^{229} .15$ There are various other examples of physical constants, such as gravitation or strong interaction, being just as life needs them to be. ${ }^{16}$ However, the point on which all fine-tuning examples converge is the strikingly low probability of the universe being exactly as life needs it to be.

Naturally, fine-tuning arguments have inspired a number of counter-arguments. Some have appealed to an 'anthropic principle' to argue that the existence of life in our universe is not at all surprising (if our universe was not life-permitting, there would be nobody to wonder about the fact that the universe is life-permitting); others have argued that mathematical probability distributions are undefined over infinitely large space of possible outcomes (i.e. possible universes). ${ }^{17}$ It has also been argued that there is no reason to believe that science will not find a natural explanation for fine-tuning, just as it managed to find natural explanations for other striking regularities in the past. ${ }^{18}$ Finally, some have suggested that the universe is in fact a multiverse, consisting of vastly many or even infinitely many universes, which would increase the probability of there being one life-permitting universe significantly. ${ }^{19}$

I will not enter these specific debates. Rather, I am interested in the question whether or not there is an in principle reason to be sceptical about the legitimacy of theistic explanations of empirical facts. To answer this question, I will now investigate whether God can be argued to play an indispensable programming role in the explanation of cosmic fine-tuning that is analogous to the role of mathematics in the explanation of other empirical regularities. If a case can be made that God does play such a role, then it seems at least prima facie legitimate to posit the existence of God as an explanation of cosmic fine-tuning. However, if such a case cannot be made, then this could be argued to constitute an in principle reason to be sceptical about theistic explanations of empirical regularities.

\footnotetext{
${ }^{15}$ See Smolin (1999, p. 45): 'In my opinion, a probability this tiny is not something we can let go unexplained. Luck will certainly not do here; we need some rational explanation of how something this unlikely turned out to be the case.' Importantly, though, Smolin does not think that the required rational explanation will feature an intelligent creator (Huberman 2006, p. 282).

${ }^{16}$ Cf. Collins (2003).

${ }^{17}$ e.g. McGrew et al. (2001)

${ }^{18}$ e.g. Harnik et al. (2006)

${ }^{19}$ e.g. Kraay (2014)
} 


\section{Does God 'program' Cosmic Fine-Tuning?}

Recall the examples of mathematical explanations introduced above. What distinguishes the honeycombs, the Magicicadas, and the sunflower seeds is the genuinely explanatory contribution of the mathematics featuring in the explanation. For simplicity, let's stick to one of the examples, say, the honeycombs.

On the face of it, there are two elements to the explanation of the phenomenon of hexagonal honeycombs. The first element is purely causal: every cell in a honeycomb exists because one or several bees extracted wax from their abdomens, manipulated it with their antennae, mandibles, or legs, and finally built it. In other words, every honeycomb exists due to a causal chain of physical events leading up from a group of bees manipulating wax to the finished honeycomb. However, the purely causal explanation leaves a crucial question open, namely, the question why the cells of the honeycomb have their striking hexagonal shape. In order to answer this question, the explanation needs to be supplemented.

The second element of the explanation, then, is purely mathematical: the most efficient way to divide a plane into regions of equal area with least total perimeter is by dividing it into regular hexagons. The geometrical properties of hexagons thus add the information needed (in addition to Darwinian explanations concerning the survival of the fittest etc.) to explain the striking shape of honeycomb shells, which is missing in the purely causal explanation. ${ }^{20}$

Let's now look at the case of fine-tuning arguments for theism. The phenomenon calling for explanation is the precise attunement of physical constants, such that life becomes possible. For every 'ordinary' physical event-a hurricane, a supernova, an atomic fission-there arguably exists a purely causal explanation involving a chain of prior physical events leading up to it. The theory of the evolution of species by random mutation and natural selection can even account for much of the intricate functional organisation of organisms on an individual as well as on a collective level. However, the physical phenomenon of, say, the cosmological constant $\Lambda$ having the value it has cannot be explained by a purely causal story; it is a brute fact. Again, the purely causal explanation leaves a crucial question open, namely, why $\Lambda$ has precisely the value necessary for the existence of life. In this case, the gap in the explanation cannot be filled mathematically; there is no theorem that can account for the value of $\Lambda$. Nevertheless, without an explanation of the value of $\Lambda$, our explanation of the apparent fine-tuning of the physical cosmos is incomplete.

Recall that in the case of the honeycombs, a mathematical theorem is put forth as a meta-natural explanation in order to complement the purely causal explanation; the argument from the explanatory indispensability of mathematics to our best scientific theories is then argued to support mathematical realism.

At this point, two questions arise:

\footnotetext{
${ }^{20}$ In fact, these two elements neither suffice to make the explanation complete. Notice that the additional information the mathematical element provides is only relevant once we establish two additional connecting claims, namely that (a) the survival of species is contingent on their fitness and efficiency, and (b) survival is what all living organisms strive for. Only with this extra-pieces of information in place does the explanation become complete.
} 
1. Is there a meta-natural hypothesis that can explain the value of $\Lambda$ and thus complete our explanation of the apparent fine-tuning of the physical cosmos?

2. Could such an hypothesis be argued to be explanatorily indispensable to our best scientific theories of the apparent fine-tuning of the physical cosmos?

The answer to question 1 is, of course, yes. There are numerous possible metanatural hypotheses that could be offered as an explanation of the value of $\Lambda$ (angels, demons, aliens), but the three most serious competitors are that the value of $\Lambda$ was intended by a designer capable of bringing $\Lambda$ about, that chance brought it about against the odds, or that our universe is only one of infinitely many spatiotemporally disconnected universes in which $\Lambda$ happens to have a value conducive to life.

Let's now turn to question 2: Could one of the three hypotheses be argued to be explanatorily indispensable to our best theories of fine-tuning? Recall the distinction between process and program explanations introduced above. Process explanations operate on the purely causal level by giving exact accounts of the empirical causes that led up to a particular physical event. A program explanation, on the other hand, is an explanation that refers to properties or entities that are themselves not causally efficacious, but that ensure the instantiation of a causally efficacious property or entity that is an actual cause of the explanandum. Which one of the three hypotheses, the designer-hypothesis, the chance-hypothesis, and the multiverse hypothesis, could be argued to play a programming role in the required sense?

Chance could have brought about any possible value for $\Lambda$. In particular, it could have brought about a whole range of values for $\Lambda$ that would have made life impossible. Thus, the chance-hypothesis does not guarantee the value of $\Lambda$ and cannot be argued to play a programming role analogous to the mathematical case.

The multiverse hypothesis, on the other hand, does guarantee the existence of at least one universe in which the value of $\Lambda$ is identical with the one instantiated in our actual world. This is because the multiverse hypothesis guarantees the existence of all possible universes. However, the multiverse hypothesis is not an explanation that answers our initial question (Why is our universe life-permitting?), but one that answers a slightly different question (Why is there any universe that is lifepermitting?). Moreover, in order to answer the question about the unexplained fact concerning $\Lambda$ (Why does $\Lambda$ have the value it needs to have for life to be possible?), the multiverse hypothesis posits yet another unexplained fact, i.e. the existence of infinitely many universes. Finally, the multiverse hypothesis does not explain the value of $\Lambda$ in terms of properties or entities that are themselves not causally efficacious. Rather, it explains the value of $\Lambda$ by positing more causally efficacious 'stuff', i.e. infinitely many physical universes. Hence, also the multiverse hypothesis doesn't meet the criteria for a program explanation.

So we are left with the designer hypothesis, and it seems evident that this hypothesis does indeed play a programming role. A designer, let's call her 'God', who intended $\Lambda$ to have the value it has and who is capable of bringing the value of $\Lambda$ about would ensure the instantiation of the precise value $\Lambda$ has. God, understood as 
an entity that is itself not causally efficacious, ${ }^{21}$ would thus guarantee the instantiation of the causally efficacious property in question - the value of $\Lambda$ - which, in turn, is an actual cause of the explanandum - the existence of life in our universe.

\section{Conclusion}

I have argued that meta-natural explanations of empirical facts are admissible just in case the explanation refers to an entity that, though not itself causally efficacious, guarantees the instantiation of a causally efficacious entity that is an actual cause of the regularity. I have then argued that only the theism hypothesis, but not the multiverse and the chance hypotheses, meets this basic admissibility criterion. I would now like to conclude this paper with a comment on why I believe that theism still fares somewhat worse as an explanatory hypothesis than the analogous case of mathematical realism I considered.

The reason is that the 'God-hypothesis' is extremely vague - too vague, perhaps, to play the programming role in a satisfactory way. In particular, it is far from being as precise as a mathematical theorem. Consider the mathematical case. What cries out for explanation in the honeycomb scenario is the peculiar shape of the honeycomb cells. Once we have identified their mathematical properties, we can (as it were) 'read off' the Honeycomb Theorem from the shape of the cells. Exactly the same holds in the cases of the Magicicadas and the sunflower seeds, although it is of course different mathematical facts doing the explanatory work there. In all three cases, however, ontological commitment to the mathematical properties and entities at work in a specific physical scenario can be used, with some additional argument, in order to ground a universal ontological commitment to mathematical entities.

It is not at all clear that the same holds of the theistic analogue. Consider the case of fine-tuning. What cries out for explanation in the example described above is the fact that the value of $\Lambda$ is within the range it has to be for the formation of stars to be possible, which, in turn, is a precondition for the existence of living organisms.

However, unlike in the honeycomb setting, it is not the case that there is any particular physical scenario from which we can 'read off' a theistic proposition that programs the value of $\Lambda$ in that particular scenario and that can be used to ground a 'universal' ontological commitment to God. Rather, we take a brute fact and offer an explanatory hypothesis about why that fact obtains. Yet there is no connection between the brute fact and the explanatory hypothesis that allows us to 'read off' any particular theistic proposition or 'theorem'. In other words, it is not clear which theistic proposition(s) exactly are doing the explanatory work.

Acknowledgements Many thanks to Sara Aronowitz, Nathan Aviezer, Kelly Clark, Scott Davison, Tyron Goldschmidt, Nidhal Gouessoum, Aaron Griffith, Douglas Kindschdi, Robert Koons, Jeffrey Koperski,

\footnotetext{
${ }^{21}$ Some might be worried about calling God, as per our definition of program explanations, an entity that is 'not causally efficacious.' However, the worry disappears as soon as it is made explicit that we understand causation in a purely physical sense according to which only physical entities and properties can stand in causal relations. And since God is (uncontroversially) not a physical entity, God is (equally uncontroversially) also not causally efficacious in the corresponding sense.
} 
Irem Kurtsal, Sam Lebens, Alan Love, Arash Naraghi, Sajjad Rizvi, Emil Salim, Aaron Segal, Josef Stern, Karl Svozil, Shira Weiss, and Karen Zwier for many helpful comments and discussions. Work on this project has been supported by the John Templeton Foundation and the European Commission, H2020MSCA-IF-2018, grant agreement number 846522.

Funding Open Access funding enabled and organized by Projekt DEAL.

Open Access This article is licensed under a Creative Commons Attribution 4.0 International License, which permits use, sharing, adaptation, distribution and reproduction in any medium or format, as long as you give appropriate credit to the original author(s) and the source, provide a link to the Creative Commons licence, and indicate if changes were made. The images or other third party material in this article are included in the article's Creative Commons licence, unless indicated otherwise in a credit line to the material. If material is not included in the article's Creative Commons licence and your intended use is not permitted by statutory regulation or exceeds the permitted use, you will need to obtain permission directly from the copyright holder. To view a copy of this licence, visit http://creativecommons.org/licenses/by/4.0/.

\section{References}

Baker, A. (2005). Are there genuine mathematical explanations of physical phenomena? Mind, 114, 22338.

Baker, A. (2009). Mathematical explanation in science. British Journal for the Philosophy of Science, 60, 611-633.

Baker, A. (2011). Explaining the applicability of mathematics in science. Interdisciplinary Science Reviews, 36, 255-267.

Bartha, P. (2010). By parallel reasoning the construction and evaluation of analogical arguments. New York: Oxford University Press.

Bigelow, J., \& Pargetter, R. (1987). Beyond the blank stare. Theoria, 53(2-2), 97-114.

Cicero, M.T. (1998). The nature of the gods (45 BCE). Oxford: Oxford University Press.

Collins, R. (2003). The teleological argument. In Moser, P., \& Copan, P. (Eds.) The rationality of theism (pp. 132-148). London: Routledge.

Colyvan, M. (2001). The indispensability of mathematics. New York: Oxford University Press.

Colyvan, M. (2002). Mathematics and aesthetic considerations in science. Mind, 111(441), 69-74.

Colyvan, M. (2010). There is no easy road to nominalism. Mind, 119(474), 285-306.

Darwin, C. (1859). On the origin of species. New York: P.F. Collier.

Enoch, D. (2011). Taking morality seriously: a defense of robust realism. New York: Oxford University Press.

Friederich, S. (2018). Fine-tuning. In Zalta, E.N. (Ed.) The Stanford Encyclopedia of Philosophy (Winter 2018 ed.): Metaphysics Research Lab, Stanford University.

Goles, E., Schulz, O., Markus, M. (2001). Prime number selection of cycles in a predator-prey model. Complexity, 6(4), 33-38.

Hales, T. (2001). The honeycomb conjecture. Discrete and Computational Geometry, 25(1), 1-22.

Harnik, R., Kribs, G., Perez, G. (2006). A universe without weak interactions. Physical Review D 74(3).

Holder, R.D. (2002). Fine-tuning, multiple universes and theism. Noûs, 36(2), 295-312.

Huberman, J. (2006). The quotable atheist. New York: Nation Books.

Jackson, F., \& Pettit, P. (1990). Program explanation: a general perspective. Analysis, 69(2), 107-117.

Jantzen, B. (2014). An introduction to design arguments. Cambridge: Cambridge University Press.

Kaplan, D. (1995). A problem in possible world semantics. In Sinnott-Armstrong, W., Diana, R., Asher, N. (Eds.) Modality, morality and belief: essays in honor of Ruth Barcan Marcus (pp. 41-52). Cambridge: Cambridge University Press.

Kraay, K. (2014). God and the multiverse: Scientific, philosophical and theological perspectives. London: Routledge.

Landau, E. (1958). Elementary number theory. New York: Chelsea Publishing Company.

Lewis, D. (1986). On the plurality of worlds. Oxford: Blackwell Publishing.

Lipton, P. (2004). What good is an explanation? In Cornwell, J. (Ed.) Explanations. Styles of Explanation in Science (pp. 1-21). Oxford: Oxford University Press. 
Lyon, A. (2012). Mathematical explanations of empirical facts, and mathematical realism. Australasian Journal of Philosophy, 90(3), 559-578.

Lyon, A., \& Colyvan, M. (2008). The explanatory power of phase spaces. Philosophia Mathematica, 16(2), 227-243.

McGrew, T., McGrew, L., Vestrup, E. (2001). Probabilities and the fine-tuning argument: a sceptical view. Mind, 110(440), 1027-1038.

Pincock, C. (2007). A role for mathematics in the physical sciences. Noûs, 41, 253-275.

Pincock, C. (2015). Abstract explanations in science. British Journal for the Philosophy of Science, 66, 857-882.

Plato. (1997). The republic. Indianapolis: Hackett.

Putnam, H. (1975). Mathematics matter and method. Cambridge: Cambridge University Press.

Putnam, H. (1979). What is mathematical truth? In Mathematics, Matter and Method: Philosophical Papers (pp. 60-78). Cambridge: Cambridge University Press.

Quine, W. (1981). Success and limits of mathematization. In Theories and things (pp. 148-155). Cambridge: Harvard University Press.

Ratzsch, D., \& Koperski, J. (2019). Teleological arguments for God's existence. In Zalta, E.N. (Ed.) The Stanford Encyclopedia of Philosophy (Summer 2019 ed.: Metaphysics Research Lab, Stanford University.

Sedley, D. (2007). Creationism and its critics in antiquity. Berkeley: University of California Press.

Smolin, L. (1999). The life of the cosmos. New York: Oxford University Press.

Sober, E. (2018). The design argument. Cambridge: Cambridge University Press.

Steiner, M. (1989). The application of mathematics to natural science. Journal of Philosophy, 86, 449-480.

Steiner, M. (1998). The applicability of mathematics as a philosophical problem. Cambridge: Harvard University Press.

Wigner, E. (1960). The unreasonable effectiveness of mathematics in the natural sciences. Communications on Pure and Applied Mathematics, 13, 1-14.

Publisher's Note Springer Nature remains neutral with regard to jurisdictional claims in published maps and institutional affiliations. 